\title{
Phylogeny of the Plant Salinity Tolerance Related HKT Genes
}

\author{
Sameera O. Bafeel ${ }^{1}$ \\ ${ }^{1}$ Department of Biology, College of Science, King Abdulaziz University (KAU), Jeddah, Saudi Arabia \\ Correspondence: Sameera O. Bafeel, Department of Biology, College of Science, King Abdulaziz University \\ (KAU), Jeddah, Saudi Arabia. Tel: 966-2695-2000 ext. 26383. E-mail: sbafil@kau.edu.sa
}

Received: January 9, 2013 Accepted: February 8, 2013 Online Published: March 13, 2013

doi:10.5539/ijb.v5n2p64

URL: http://dx.doi.org/10.5539/ijb.v5n2p64

\begin{abstract}
Success in the extension of crop production in the salt affected area may help to meet the demand of the world's hunger. In plants, $\mathrm{Na}+$ permeable $H K T$ (High-affinity $\mathrm{K}^{+}$Transporter) gene family represents a primary mechanism for mediating salt tolerance. Better understanding of the relationship among $H K T$ genes may provide a framework for the development of salt tolerance cultivars. In this study, phylogeny of plant- $H K T$ gene family using the available nucleotide sequences in the current databases was examined. This study showed a total of 6 phylo-groups. Phylogenetic analyses based on $H K T$ gene sequences demonstrated distinct lineages for monocots and eudicots. Group I, II and VI contained only monocots. Eudicots were clustered in group III, IV and V. A stable relationship was observed among the members of the group I (previously described as subfamily 2). Overall pairwise mean distance was very high (1.13), indicating the high level of diversity of the $H K T$ gene family. Observed pairwise distances were: Group 4 (eudicots, 0.81) > group 6 (monocots, 0.52) > group 2 (monocots, 0.35 ) $>$ group 1 (monocots, 0.33 ) $\approx$ group 5 (eudicots, 0.33 ) > group 3 (eudicots, 0.17 ).
\end{abstract}

Keywords: $H K T$, plant, phylogeny

\section{Introduction}

Total salt affected land-area was estimated as 955 million hectares and secondary build up of salts affects about 80 million hectares of land in the arid and semiarid regions (Ghassemi et al., 1995). Number of hungry people in the world is estimated as 925 million in 2010 (World Hunger Education Service, 2012). Therefore, extension of cultivation in salt affected area may help to meet the demand for food. In general, plant species are sensitive to high concentrations of sodium $\left(\mathrm{Na}^{+}\right)$, which causes combined $\mathrm{Na}^{+}$toxicity and osmotic stress. Plants have multiple $\mathrm{Na}^{+}$transport systems to bypass $\mathrm{Na}^{+}$toxicity. Essential physiological functions of major $\mathrm{Na}^{+}$ transporters and their mechanisms mediating salinity resistance have been identified in extensively studied plant Arabidopsis, including the SOS1 (Salt Overly Sensitive), AtNHX (Arabdiopsis thaliana $\mathrm{Na}^{+} / \mathrm{H}^{+}$antiporters) and AtHKT1;1 (A. thaliana High-affinity $\mathrm{K}^{+}$Transporter) gene family (Horie et al., 2009). The HKT gene-family is expressed in xylem parenchyma cells and protects leaves from salinity stress by removing sodium from the xylem-sap (Ren et al., 2005; Sunarpi et al., 2005). Therefore, xylem-parenchyma-expressed $\mathrm{Na}^{+}$permeable plant $H K T$ transporters represent a primary mechanism for mediating salt tolerance and $\mathrm{Na}^{+}$exclusion from leaves as observed in Arabidopsis and other monocot crop-plants as well (Horie et al., 2009). Nevertheless, variation in copy number for individual $H K T$ gene members was observed among the barley, wheat, and rice genomes, and among the different wheat genomes (Huang et al., 2008). HKT gene sequences are not closely related (Platten et al., 2006). Phylogenetic trees of each family define the evolutionary relationships of the members to each other. In each family, closely related isoforms and separate subfamilies of $\mathrm{K}^{+}$transporters existed, indicating possible redundancies and specialized functions (Amrutha et al., 2007). To meet this challenge of world hunger, it is important to understand the mechanisms of salt tolerance to utilize the unused salt-affected land. Better understanding of the relationship among the $H K T$ genes may provide a framework for the development of salt tolerance cultivars. Phylogenetic study on $H K T$ gene family is very limited except earlier work of Platten et al. (2006), Heidari et al. (2011) and very recently Porras et al. (2012). In this study, the phylogeny of plant-HKT genes was examined using the available nucleotide sequences in the current databases.

\section{Materials and Methods}

The Entrez Nucleotides database (http://www.ncbi.nlm.nih.gov/) is a collection of nucleotide sequences from GenBank, RefSeq, and PDB (Protein Data Bank). Sodium transporter (HKT) gene-related nucleotide sequences 
of plants were retrieved from the database and aligned using Clastal X (version 2.0). The evolutionary history of the $H K T$ gene was inferred by using the Maximum Likelihood (ML) and Maximum Parsimony (MP) methods. Evolutionary analyses were conducted in MEGA5 (Tamura et al., 2011). The bootstrap consensus tree inferred from 1000 replicates was taken to represent the evolutionary history of the $H K T$-related genes that were included in the analyses (Felsenstein, 1985). The percentage of replicate trees in which the associated taxa clustered together in the bootstrap test (1000 replicates) was shown next to the branches (Tamura \& Nei, 1993). The trees were drawn to scale, with branch lengths measured in the number of substitutions per site. The analysis involved a total of 32 nucleotide sequences. Codon positions included were 1 st +2 nd +3 rd + noncoding. All positions containing gaps and missing data were eliminated by selecting the complete-deletion under the gaps/missing data treatment option. There were a total of 229 nucleotide positions in the final dataset after the elimination of gaps and missing data. Estimates of the overall mean evolutionary divergence of the sequence-pairs were conducted using the maximum composite likelihood model (Tamura et al., 2004) using MEGA5 (Tamura et al., 2011).

\section{Results and Discussions}

$H K T$ gene-related sequences currently present in the database were mainly from monocots ( $82 \%)$ and only $18 \%$ from eudicots (Figure 1). The phylogenetic tree inferred by using the Maximum Likelihood (ML) method showed a total of 6 phylo-groups of HKT-related genes with high bootstrap supports (65-100\%) (Figure 2). Phylogenetic tree constructed using Maximum Parsimony (MP) method also supported this grouping, however, with less bootstrap support for the groups IV, and VI. There was a distinct grouping for monocots and eudicots. Group I contained only the monocots, which included Oryza sativa (Poaceae), Triticum aestivum (Triticeae), Hordeum vulgare (Triticeae) and Phragmites australis (Poaceae) (Figures 2 and 3). Clustering of monocots in group I was supported by very high bootstrap value $(100 \%, 97 \%$; Figures 2 and 3$)$. None of the members of the eudicots was observed in this clade. Plant species belonging to monocots (Oryza sativa, Poaceae) were also placed in group II and group VI. In contrast to HKT genes found in rice (group I), $H K T$ genes in group II, and VI (rice) seem to be more diverse as observed by previous study as well (Platten et al., 2006). Earlier work of Platten et al. (2006) proposed nomenclature for the HKT gene family and showed that the gene family splits into two major clades; subfamily 1 , and subfamily 2 . Nomenclature was described according to the species $H K T$ subfamily, and number of genes within a species (TaHKT2;1, OsHKT2;3 etc.). Nonetheless, the proposed unified nomenclature cannot be applied to all plant $H K T$ genes (Porras et al., 2012). Diversity within the HKT transporter family might be central to the regulation of $\mathrm{K}^{+}$and $\mathrm{Na}^{+}$accumulation (Jabnoune et al., 2009). Platten et al. (2006) noted that all HKT genes contain two introns, these are significantly larger in members of the rice groups (groups II, and VI) compared with the members of the group I (Figures 2 and 3). Two isoforms of $H K T$ transporters, a Na ${ }^{+}$transporter $(O s H K T 1)$ and a $\mathrm{Na}^{+}$and $\mathrm{K}^{+}$coupled transporter (OsHKT2), may contribute for such heterogeneity in the salt tolerant rice (Horie et al., 2001). Study of HKT-gene in Gramineae via in silico cDNA-AFLP analysis showed that plants clustered in four groups. Group 1 consisted of 4 plants (Secale cereale, T. monococum, T. turgidum, and Aegilops tauschii), group 2 consisted of 1 plant (Avena sativa), group 3 consisted of 1 plant (Oryza sativa japonica) and group 4 consisted of 2 plants (H. vulgare and T. aestivum) (Heidari et al., 2011). Variation in copy number for individual HKT gene members was observed between the barley, wheat and rice genomes and even between the different wheat genomes (Huang et al., 2008). This study showed $A$. thaliana HKT gene was placed in group IV separated from $O$. sativa or T. aestivum. Comparative analysis of rice $\mathrm{K}+$ channels also revealed that Arabidopsis is quite distant from rice (Amrutha et al., 2007). There was very high sequence variation among $H K T$-related genes (overall pairwise mean distance was 1.13 ). Group wise distances were observed as group $4(0.81)>$ group $6(0.52)>$ group $2(0.35)>$ group $1(0.33) \approx$ group $5(0.33)>$ group $3(0.17)$. The independent multiplication of $H K T$-coding genes may be correlated with the affinity of the vascular plants to the particular environments. Rice $H K T$ gene family was very heterogeneous and clustered in 3 different groups (Groups I, III and VI; Figures 2 and 3). Probably, a larger variety of $\mathrm{Na}^{+} / \mathrm{K}^{+}$ transporters provides some advantage for better adaptation (Porras et al., 2012). For instance in rice, OsHKT2;1 is known to be permeable to both $\mathrm{Na}^{+}$and $\mathrm{K}^{+}$and to be able to mediate symport activity in some conditions (Jabnoune et al., 2009), and this functional group is clustered in group I. Nevertheless, OsHKT1;1 and OsHKT1;3 are shown to be permeable to $\mathrm{Na}^{+}$only (Jabnoune et al., 2009) and this functional type is clustered together (separate from $O s H K T 2 ; 1)$ in group VI. 


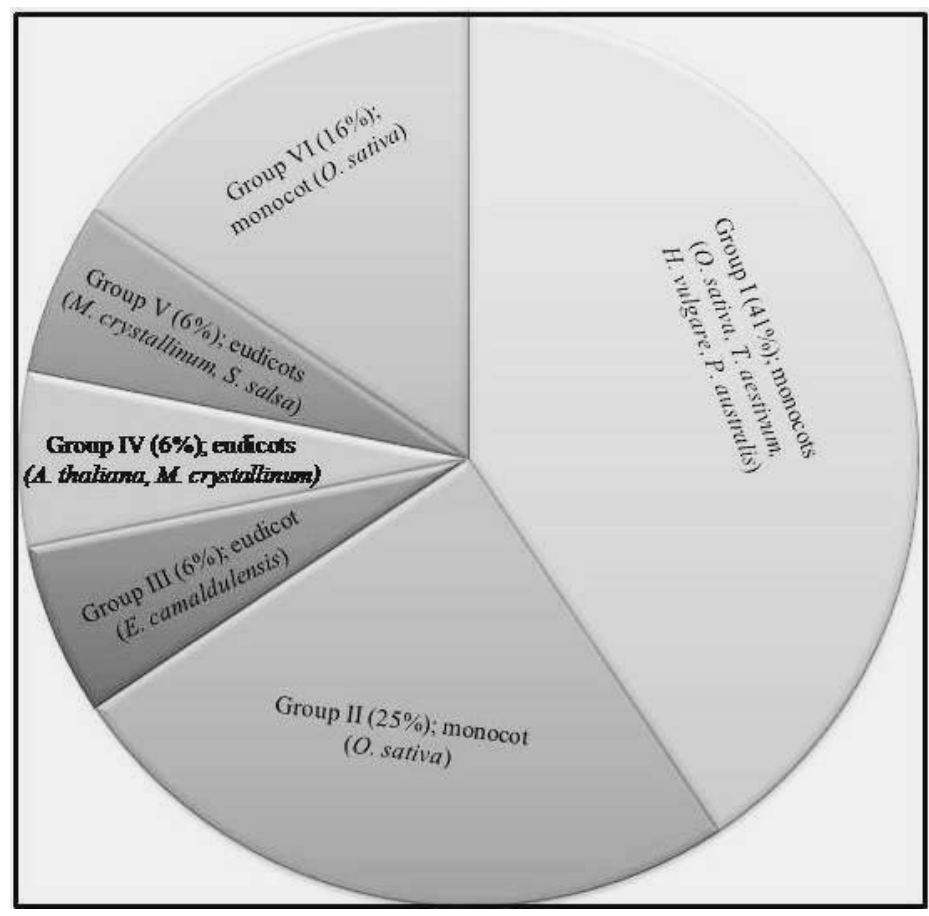

Figure 1. Distribution of the members of the $H K T$ gene family included in this study

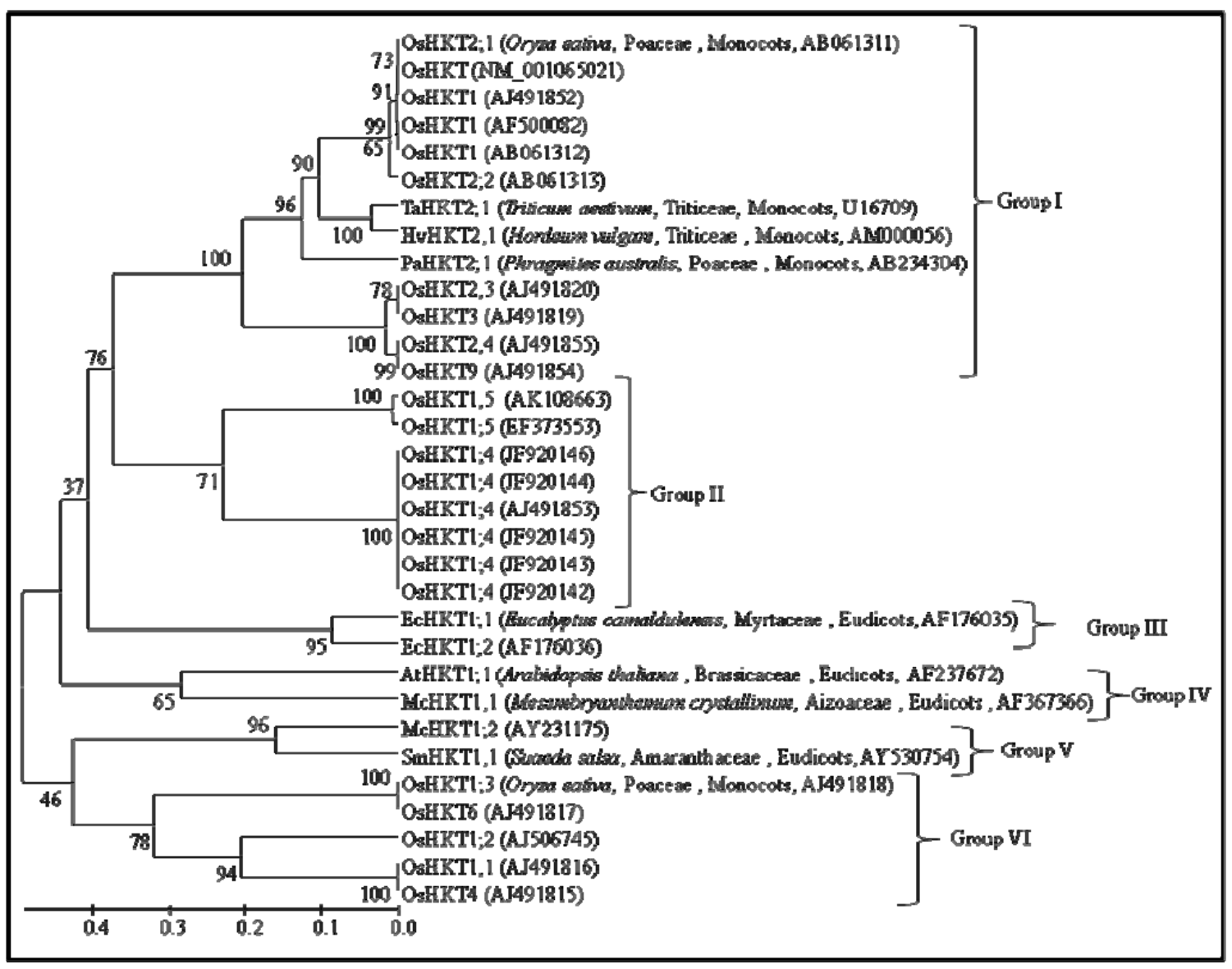

Figure 2. Maximum likelihood (ML) tree showing the relationship of the $H K T$ gene family. Species name, family, monocot/eudicot, and EMBL/DDBJ/GenBank accession numbers are given in the parentheses 


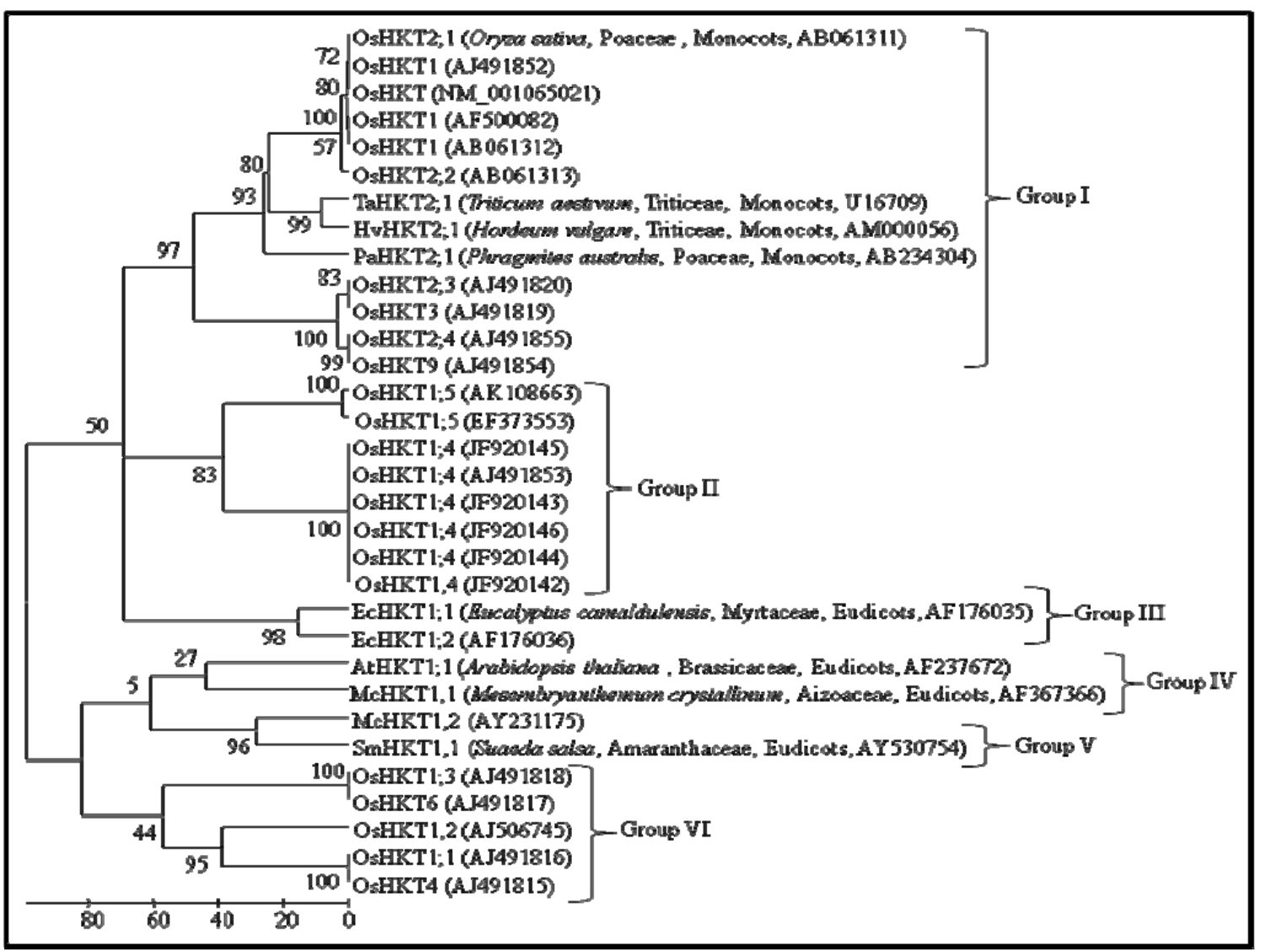

Figure 3. Maximum parsimony (MP) tree showing the relationship of the $H K T$ gene family. Species name, family, monocot/eudicot, and EMBL/DDBJ/GenBank accession numbers are given in the parentheses

High divergence of HKT proteins raises questions about the evolutionary changes in the function of the protein family. Recent study showed that $H K T$ family members are not only sodium-selective uniporters or sodium-potassium symporters; the rice gene OsHKT2;4 protein also functions as a $\mathrm{Ca}^{2+}$ permeable cation channel that conducts current carried by a wide range of monovalent and divalent cations (Lan et al., 2010).

A total of $229 \mathrm{nt}$ positions was used in this study, after the elimination of gaps and missing data of $H K T$ gene in the final dataset. Platten et al. (2006) included nearly complete sequences of $H K T$ genes. Similar to this study, Platten et al. (2006) also observed very stable relationship for the members of the group 1 (subfamily 2). Splitting of the members of the subfamily 1, as shown by this study (groups II-VI), was predicted by Platten et al. (2006) as well. Splitting pattern was noted for the HKT-related genes from Selaginella moellendorffii and Physcomitrella patens (Porras et al., 2012). This study showed very high variability of the HKT-related genes. Availability of the $H K T$ gene-related expressed protein sequences may clarify further relationships in the near future.

\section{References}

Amrutha, R. N., Sekhar, P. N., Varshney,R. K., \& Kishor, P. B. K. (2007). Genome-wide analysis and identification of genes related to potassium transporter families in rice (Oryza sativa L.). Plant Science, 172(4), 708-721. http://dx.doi.org/10.1016/j.plantsci.2006.11.019

Felsenstein, J. (1985). Confidence limits on phylogenies: An approach using the bootstrap. Evolution, 39, 783-791. Retrieved from http://www.jstor.org/stable/2408678

Ghassemi, F., Jakeman A. J., \& Nix, H. A. (1995). Salinisation of land and water resources: Human causes, extent, management and case studies. Sydney, Australia, and CAB International, Wallingford: UNSW Press. UK. 
Heidari, P., Falaknaz, M., Mehrabi, A. A., Kahrizi, D., \& Yari, K. (2011). Phylogenetic study of HKT-gene in Gramineae via insilico cDNA-AFLP analysis. American Journal of Scientific Research, 19, 6-12. Retrieved January 04, 2013, from http://www.eurojournals.com/AJSR_19_01.pdf

Horie, T., Hauser, F., \& Schroed, J. I. (2009). HKT transporter-mediated salinity resistance mechanisms in Arabidopsis and monocot crop plants. Trends in Plant Science, 14(12), 660-668. http://dx.doi.org/10.1016/j.tplants.2009.08.009.

Horie, T., Yoshida, K., Nakayama, H., Yamada, K., Oiki, S., \& Shinmyo, A. (2001). Two types of HKT transporters with different properties of $\mathrm{Na}^{+}$and $\mathrm{K}^{+}$transport in Oryza sativa. The Plant Journal, 27(2), 129-38. http://dx.doi.org/10.1046/j.1365-313x.2001.01077.x

Huang, S., Spielmeyer, W., Lagudah, E. S., \& Munns, R. (2008). Comparative mapping of $H K T$ genes in wheat, barley and rice, key determinants of $\mathrm{Na}+$ transport, and salt tolerance. Journal of Experimental Botany, 59(4), 927-37. http://dx.doi.org/10.1093/jxb/ern033

Jabnoune, M., Espeout, S., Mieulet, D., Fizames, C., Verdeil, J. C., Conéjéro, G., ... Véry, A. A. (2009). Diversity in expression patterns and functional properties in the rice $H K T$ transporter family. Plant Physiology, 150(4), 1955-1971. http://dx.doi.org/10.1104/pp.109

Lan, W. Z., Wang, W., Wang, S. M., Li, L. G., Buchanan, B. B., Lin, H. X., ... Luan, S. (2010). A rice high-affinity potassium transporter $(H K T)$ conceals a calcium-permeable cation channel. Proceedings of the National Academy of Sciences (USA), 107(15), 7089-7094. http//dx.doi.org/ 10.1073/pnas.1000698107

Platten, D., Cotsaftis, O., Berthomieu, P., Bohnert, H., Davenport, R. J., Fairbairn, D. J., ... Tester, M. (2006). Nomenclature for HKT transporters, key determinants of plant salinity tolerance. Trends in Plant Science, 11(8), 372-374. http://dx.doi.org/10.1016/j.tplants.2006.06.001

Porras, J. L. G., Pachón, D. M. R., Benito, B., \& Haro, R. (2012). Phylogenetic analysis of $\mathrm{K}^{+}$transporters in bryophytes, lycophytes, and flowering plants indicates a specialization of vascular plants. Frontiers in Plant Science, 3, 167. http//dx.doi.org/10.3389/fpls.2012.00167

Ren, Z. H., Gao, J. P., Li, L. G., Cai, X. L., Huang, W., Chao, D. Y., ... H. X. (2005). A rice quantitative trait locus for salt tolerance encodes a sodium transporter. Nature Genetics, 37(10), 1141-1146. http//dx.doi.org/10.1038/ng1643

Sunarpi, H. T., Motoda, J., Kubo, M., Yang, H., Yoda, K., Horie, R., ... Uozumi, N. (2005). Enhanced salt tolerance mediated by AtHKT1 transporter-induced $\mathrm{Na}+$ unloading from xylem vessels to xylem parenchyma cells. The Plant Journal, 44, 928-938. http//dx.doi.org/10.1111/j.1365-313X.2005.02595.x

Tamura, K., Nei, M., \& Kumar, S. (2004). Prospects for inferring very large phylogenies by using the neighbor-joining method. Proceedings of the National Academy of Sciences (USA), 101, 11030-11035. http//dx.doi.org/10.1073/pnas.0404206101

Tamura, K., \& Nei, M. (1993). Estimation of the number of nucleotide substitutions in the control region of mitochondrial DNA in humans and chimpanzees. Molecular Biology and Evolution, 10(3), 512-526. Retrieved January 02, 2013, from http://mbe.oxfordjournals.org/content/10/3/512.long

Tamura, K., Peterson, D., Peterson, N., Stecher, G., Nei, M., \& Kumar, S. (2011). MEGA5: Molecular evolutionary genetics analysis using maximum likelihood, evolutionary distance and maximum parsimony methods. Molecular Biology and Evolution, 28, 2731-2739. http//dx.doi.org/ 10.1093/molbev/msr121

World Hunger Education Service. (2012). 2012 World Hunger and Poverty Facts and Statistics. Retrieved from http://www.worldhunger.org/articles/Learn/world\%20hunger\%20facts\%202002.htm 